Research Article

\title{
Synthesis, Spectral Characterization, and In Vitro Cytotoxicity of Some Fe(III) Complexes Bearing Unsymmetrical Salen-Type Ligands Derived from 2-Hydroxynaphthaldehyde and Substituted Salicylaldehydes
}

\author{
Quang Trung Nguyen (D, Phuong Nam Pham Thi, and Nguyen Van Tuyen \\ Institute of Chemistry, Vietnam Academy of Science and Technology, 18 Hoang Quoc Viet,Cau Giay, Hanoi, Vietnam \\ Correspondence should be addressed to Quang Trung Nguyen; trungquang_cnhh@yahoo.com
}

Received 15 April 2021; Revised 9 June 2021; Accepted 11 June 2021; Published 19 June 2021

Academic Editor: Damião Pergentino de Sousa

Copyright (C) 2021 Quang Trung Nguyen et al. This is an open access article distributed under the Creative Commons Attribution License, which permits unrestricted use, distribution, and reproduction in any medium, provided the original work is properly cited.

\begin{abstract}
Six Fe(III) complexes bearing unsymmetrical salen-type ligands derived from 2-hydroxynaphthaldehyde and substituted salicylaldehydes were synthesized by coordinating the unsymmetrical salen-type ligands with $\mathrm{FeCl}_{3} \cdot 6 \mathrm{H}_{2} \mathrm{O}$. The synthetic complexes were characterized by electrospray ionization mass spectra (ESI-MS), effective magnetic moments $\left(\mu_{\mathrm{eff}}\right)$, and infrared (IR) and ultraviolet-visible (UV-Vis) spectra. The spectroscopic data are in good agreement with the suggested molecular formulae of the complexes. Their cyclic voltammetric studies in acetonitrile solutions showed that the $\mathrm{Fe}(\mathrm{III}) / \mathrm{Fe}$ (II) reduction processes are electrochemically irreversible. The in vitro cytotoxicity of the obtained complexes was screened on human cancer cell lines KB (a subline of Hela tumor cell line) and HepG2 (a human liver cancer cell line) and a normal human cell line HEK-293 (Human Embryonic Kidney cell line). The results showed that the synthetic Fe(III) complexes are highly cytotoxic and quite selective. The synthetic complexes bearing unsymmetrical salen-type ligands with different substituted groups in the salicyl ring indicate different cytotoxicity.
\end{abstract}

\section{Introduction}

The developments in transition metal complexes have gained considerable attention about various structures and potential applications in catalysis, analysis, advanced materials science, and biochemistry especially [1-9]. Besides the meaningful efficiency of platinum complexes as anticancer agents [10-12], recent bioinorganic chemists have focused on the design and preparation of new transition metal complexes with Schiff base ligands [13-15]. Schiff bases with donors ( $\mathrm{N}, \mathrm{O}$, etc.) have been widely investigated due to their diverse pharmacological applications [16], in which tetradentate Schiff bases are derived from salicylaldehydes and diamines, which form the Schiff bases known as "salen" with an $\mathrm{N} 2 \mathrm{O} 2$ donor group being able to coordinate with different metal ions [17]. These diamine Schiff bases with $\mathrm{OH}$ groups in ortho positions are of interest because of the presence of tautomerism between keto-amine and enolimine forms [18]. The transition metal complexes of tetradentate Schiff bases have received much attention about their structure, magnetic and electrochemical characterization, and their potential application in biological functions lately. They predominantly show their antiproliferative, antimalarial, antifungal, antipyretic, and antidiabetic activities $[19,20]$. Besides, many symmetrical tetradentate Schiff bases and their transition metal complexes have been extensively studied on the preparation, spectral characterization, and biological activity [21-23]; recently, unsymmetrical tetradentate Schiff base ligands and their complexes have been paid attention [24-26]. It should be realized that the coordinated ligands around central metal ions in natural systems are unsymmetrical. Therefore, in this work, we continue with the synthesis, spectral characterization, and in vitro anticancer behavior of Fe(III) complexes 
bearing unsymmetrical salen-type Schiff bases derived from 2-hydroxynaphthaldehyde and substituted salicylaldehydes.

\section{Materials and Methods}

Chemical reagents used in the present study, such as 0 phenylenediamine (98\%), 2-hydroxy-1-naphthaldehyde (tech.), and salicylaldehydes, were obtained from Across Organics and used without further purification. All solvents were distilled following the laboratory procedures before use.

Ultrahigh-performance liquid chromatography combined with hydride quadrupole time-of-flight tandem mass spectra (HP-TOF-MS) of the synthetic unsymmetrical tetradentate Schiff base ligands was conducted on an ExionLC AC Series HPLC system coupled with a hybrid quadrupole time-of-flight tandem mass spectrometer (X500R QTOF System) equipped with TurbolonSpray source. Chromatographic separation was performed on a Kinetex $\mathrm{C}_{18}$ column $(30 \mathrm{~mm} \times 2.1 \mathrm{~mm}, 1.7 \mu \mathrm{m})$, and the column temperature was maintained at $30^{\circ} \mathrm{C}$. The mobile phase consisted of methanol and water containing $0.1 \%$ formic acid in a gradient mode of $50 \%$ methanol for $0-5 \mathrm{~min}$ and $100 \%$ methanol at $5 \mathrm{~min}$ with

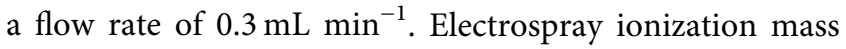
spectra (ESI-MS) $(\mathrm{m} / z)$ were recorded on Agilent 6310 Ion Trap spectrometer. Infrared spectra (IR, 4000-400 $\mathrm{cm}^{-1}$ ) were carried out on a PerkinElmer Spectrum Two spectrophotometer using a $\mathrm{KBr}$ pellet. ${ }^{1} \mathrm{H}-\mathrm{NMR}$ and ${ }^{13} \mathrm{C}-\mathrm{NMR}$ spectra were determined in $\mathrm{DMSO}-\mathrm{d}_{6}$ solution using a Bruker Advance $500 \mathrm{MHz}$ NMR spectrometer with TMS as the internal standard and chemical shifts $(\delta)$ recorded in ppm. UV-Visible absorption spectra of the synthetic compounds $(200-600 \mathrm{~nm})$ were estimated in methanol solution $\left(3 \times 10^{-5} \mathrm{M}\right)$ with PerkinElmer Lambda UV-35 spectrophotometer. Magnetic susceptibility measurements of synthetic $\mathrm{Fe}$ (III) complexes were determined at room temperature using a magnetic susceptibility balance (Mark 1, serial No. 25179) of Sherwood Scientific, Ltd.

2.1. Synthesis of Unsymmetrical Salen-Type Schiff Base Ligands. Unsymmetrical salen-type Schiff base ligands were prepared following a two-step procedure similar to the known procedure $[27,28]$. In the first step, monocondensed half-units were prepared by the condensation of $o$-phenylenediamine with 2-hydroxy-1-naphthaldehyde. In the second step, the monocondensed half-unit was mixed with an ethanol solution of relative salicylaldehydes. O-Phenylenediamine $(15.4 \mathrm{mmol})$ dissolved in ethanol $(20 \mathrm{~mL})$ was added in a $100 \mathrm{ml}$ flask containing 2-hydroxy-1-naphthaldehyde $(15.5 \mathrm{mmol})$ in ethanol $(15 \mathrm{~mL})$ and was stirred for $3 \mathrm{~h}$. After the monocondensed half-units were obtained completely by TLC checking, relative salicylaldehyde $(15.5 \mathrm{mmol})$ in ethanol $(15 \mathrm{~mL})$ was added and the new mixture was put in an ultrasonic case, Hwashin Power Sonic 405 , for $1 \mathrm{~h}$. Then, the productive precipitates were collected after being filtered and washed by cold ethanol. The obtained products are soluble in DMSO, methanol, dichloromethane, and ethyl acetate. The products were recrystallized from ethyl acetate and dried in vacuo.

(Z)-1-(((2-((E)-(2-Hydroxybenzylidene) amino) phenyl) amino) methylene) naphthalen-2 (1H)-one $\left(\mathrm{H}_{2} \mathrm{~L} 1\right)$ : yellow powder, 91\%; HP-TOF-MS (m/z): $367.1430[\mathrm{M}+\mathrm{H}]^{+}$ (Cal. 367.4199); IR (KBr, cm $\left.{ }^{-1}\right): 3060(\nu, \mathrm{C}-\mathrm{H}), 2678(\nu$, $\mathrm{O}-\mathrm{H}), 1611(\nu, C=N), 1571(\nu, C=C), 1483,1353,1315$ and $1278(\nu, \mathrm{C}-\mathrm{N}), 1188$ and $1153(\nu, \mathrm{C}-\mathrm{O}) ; 837,744(\delta, \mathrm{C}-\mathrm{H})$, 481; ${ }^{1}$ H-NMR (DMSO-d $\left.6,500 \mathrm{MHz}, \delta(\mathrm{ppm}), J(\mathrm{~Hz})\right): \delta$ $15.63(\mathrm{~d}, J=7.0,1 \mathrm{H}, \mathrm{NH}), 11.93(\mathrm{~s}, 1 \mathrm{H}, \mathrm{OH}), 9.60$ (d, $J=7.0$, $1 \mathrm{H}, \mathrm{HC}-\mathrm{N}), 8.99(\mathrm{~s}, 1 \mathrm{H}, \mathrm{HC}=N), 8.43(\mathrm{~d}, J=8.5,1 \mathrm{H}, \mathrm{Naph})$, 7.99 (d, $J=8.5,1 \mathrm{H}, \mathrm{Naph}), 7.91$ (d, $J=7.5,1 \mathrm{H}, \mathrm{Sal}), 7.86$ (d, $J=9.5,1 \mathrm{H}, \mathrm{Naph}), 7.73$ (d, $J=6.5,1 \mathrm{H}, \mathrm{Ph}), 7.51$ (t, $J=7.0$, 1H, Naph), 7.44 (m, 3H, 1H-Naph, 2H-Sal), 7.36 (t, J=7.5, $1 \mathrm{H}, \mathrm{Ph}), 7.31(\mathrm{t}, J=7.0,1 \mathrm{H}, \mathrm{Ph}), 7.00(\mathrm{~m}, 2 \mathrm{H}, 1 \mathrm{H}-\mathrm{Ph}$, 1H-Sal), 6.90 (d, $J=9.5,1 \mathrm{H}, \mathrm{Naph}) ;{ }^{13} \mathrm{C}-\mathrm{NMR}$ (DMSO-d ${ }_{6}$, $125 \mathrm{MHz}, \delta(\mathrm{ppm})): \delta 174.31(1 \mathrm{C}, C=O), 162.98(1 \mathrm{C}, C=N)$, 159.70 (1C, C-O), 152.68 (1C, HC-NH), $141.26\left(1 \mathrm{C}, \mathrm{N}-\mathrm{C}_{\mathrm{Ar}}\right.$ ), 137.69 (1C, HN-C Ar ), 136.60 (1C, Naph), 133.63 (1C, Naph), 133.47 (1C, Sal), 131.80 (1C, Sal), 128.99 (1C, Naph), 128.14 (1C, Naph), 127.71 (1C, Naph), 126.95 (1C, Naph), 126.31 (1C, Ph), 123.57 (1C, Naph), 123.41 (1C, Ph), 120.21 (1C, Ph), 120.11 (1C, Naph), 119.54 (1C, Naph), 119.32 (1C, Sal), 118.26 (1C, Sal), 116.62 (1C, Sal), 108.51 (1C, Ph); UV-Vis $\left(\mathrm{MeOH}, 3 \times 10^{-5} \mathrm{M}, \lambda / \mathrm{nm}, \varepsilon / \mathrm{cm}^{-1} \mathrm{M}^{-1}\right): 233(42,000), 266$ (22,333), 320 (17,000), 348 (16,333), 450 (12,333), 472 $(11,333)$.

(Z)-1-(((2-((E)-(5-Fluoro-2-hydroxybenzylidene) amino) phenyl) amino) methylene) naphthalen-2 (1H)-one $\left(\mathrm{H}_{2} \mathrm{~L} 2\right)$ : yellow powder, 93\%; HP-TOF-MS $(\mathrm{m} / z): 385.1379$ $[\mathrm{M}+\mathrm{H}]^{+}(\mathrm{Cal} .385 .4104)$; IR $\left(\mathrm{KBr}, \mathrm{cm}^{-1}\right): 2919(\nu, \mathrm{C}-\mathrm{H})$, $2672(\nu, \mathrm{O}-\mathrm{H}), 1618(\nu, C=N), 1576(\nu, C=C), 1485,1352$, 1313 and $1274(\nu, \mathrm{C}-\mathrm{N}), 1199$ and $1140(\nu, \mathrm{C}-\mathrm{O}) ; 824,745(\delta$, C-H), 478; ${ }^{1}$ H-NMR (DMSO-d 6 , $500 \mathrm{MHz}, \delta(\mathrm{ppm}), J(\mathrm{~Hz})$ ): $\delta 15.69(\mathrm{~d}, J=8.5,1 \mathrm{H}, \mathrm{NH}), 11.36(\mathrm{~s}, 1 \mathrm{H}, \mathrm{OH}), 9.57(\mathrm{~d}$, $J=8.0,1 \mathrm{H}, \mathrm{HC}-\mathrm{N}), 8.99(\mathrm{~s}, 1 \mathrm{H}, \mathrm{HC}=N), 8.42(\mathrm{~d}, J=8.5,1 \mathrm{H}$, Naph), 8.04 (d, $J=8.5,1 \mathrm{H}, \mathrm{Naph}), 7.92$ (dd, $J=9.0,3.5,1 \mathrm{H}$, Sal), 7.85 (d, $J=9.0,1 \mathrm{H}, \mathrm{Naph}), 7.71$ (d, $J=7.0,1 \mathrm{H}, \mathrm{Ph}), 7.50$ (t, J=7.0, 1H, Naph), 7.44 (m, 2H, 1H-Naph, 1H-Sal), 7.35 (t, J= 7.5, 1H, Ph), 7.29 (m, 2H, Ph), 7.00 (q, 1H, Sal), 6.85 (d, $J=9.5,1 \mathrm{H}, \mathrm{Naph}) ;{ }^{13} \mathrm{C}-\mathrm{NMR}\left(\mathrm{DMSO}_{-}, \mathrm{d}_{6}, 125 \mathrm{MHz}, \delta\right.$ $(\mathrm{ppm})): \delta 175.81(1 \mathrm{C}, C=O), 159.79(1 \mathrm{C}, C=N), 156.29$ and 155.72 (1C, C-O), 154.42 (1C, C-F), 151.44 (1C, HC-NH), $140.79\left(1 \mathrm{C}, \mathrm{N}-\mathrm{C}_{\mathrm{Ar}}\right), 138.02\left(1 \mathrm{C}, \mathrm{HN}-\mathrm{C}_{\mathrm{Ar}}\right), 136.30(1 \mathrm{C}$, Naph), 133.65 (1C, Naph), 129.03 (1C, Naph), 128.19 (1C, Naph), 128.00 (1C, Naph), 126.80 and 126.22 (1C, Sal), 124.20 (1C, Naph), 123.41 (1C, Naph), 121.39 (1C, Naph), 121.33 (1C, Ph), 120.57 and 120.38 (1C, Sal), 120.05 (1C, Ph), 119.05 (1C, Ph), 118.09 and 118.02 (1C, Sal), 117.86 (1C, Naph), 115.56 and 115.37 (1C, Sal), 108.41 (1C, Ph); UV-Vis $\left(\mathrm{MeOH}, 3 \times 10^{-5} \mathrm{M}, \lambda / \mathrm{nm}, \mathcal{\varepsilon} / \mathrm{cm}^{-1} \mathrm{M}^{-1}\right): 234$ (40,333), 265 $(21,667), 320(15,000), 355(16,000), 450(12,000), 472$ $(11,333)$.

(Z)-1-(((2-((E)-(5-Chloro-2-hydroxybenzylidene) amino) phenyl) amino) methylene) naphthalen-2 (1H)-one $\left(\mathrm{H}_{2} \mathrm{~L} 3\right)$ : yellow powder, 93\%; HP-TOF-MS $(\mathrm{m} / z): 401.1046$ $[\mathrm{M}+\mathrm{H}]^{+}$(Cal. 401.8647); IR $\left(\mathrm{KBr}, \mathrm{cm}^{-1}\right): 3060(\nu, \mathrm{C}-\mathrm{H})$, 2612 ( $\nu, \mathrm{O}-\mathrm{H}), 1618(\nu, C=N), 1586(\nu, C=C), 1477,1352$, 1314 and $1278(\nu, \mathrm{C}-\mathrm{N}), 1180(\nu, \mathrm{C}-\mathrm{O}) ; 828,749(\delta, \mathrm{C}-\mathrm{H})$, 
488; ${ }^{1}$ H-NMR (DMSO-d $\left.\mathrm{d}_{6}, 500 \mathrm{MHz}, \delta(\mathrm{ppm}), J(\mathrm{~Hz})\right): \delta$ $15.72(\mathrm{~d}, J=8.0,1 \mathrm{H}, \mathrm{NH}), 11.72(\mathrm{~s}, 1 \mathrm{H}, \mathrm{OH}), 9.58(\mathrm{~d}, J=7.0$, $1 \mathrm{H}, \mathrm{HC}-\mathrm{N}), 8.99(\mathrm{~s}, 1 \mathrm{H}, \mathrm{HC}=\mathrm{N}), 8.44(\mathrm{~d}, J=8.0,1 \mathrm{H}, \mathrm{Naph})$, 8.15 (d, $J=3.0,1 \mathrm{H}, \mathrm{Sal}), 8.04$ (d, $J=8.0,1 \mathrm{H}, \mathrm{Naph}), 7.85$ (d, $J=9.0,1 \mathrm{H}, \mathrm{Naph}), 7.72(\mathrm{~d}, J=7.0,1 \mathrm{H}, \mathrm{Ph}), 7.50(\mathrm{t}, J=7.0$, 1H, Naph), 7.45 (m, 3H, 1H-Naph, 1H-Ph, 1H-Sal), 7.35 (t, $J=7.5,1 \mathrm{H}, \mathrm{Ph}), 7.31(\mathrm{t}, 1 \mathrm{H}, \mathrm{Ph}), 7.00(\mathrm{q}, 1 \mathrm{H}, \mathrm{Sal}), 6.85$ (d, $J=9.5,1 \mathrm{H}, \quad$ Naph); ${ }^{13} \mathrm{C}-\mathrm{NMR}$ (DMSO-d $\mathrm{d}_{6}, 125 \mathrm{MHz}, \delta$ $(\mathrm{ppm})): \delta 175.16(1 \mathrm{C}, C=O), 160.03(1 \mathrm{C}, C=N), 158.12(1 \mathrm{C}$, C-O), $151.90(1 \mathrm{C}, \mathrm{HC}-\mathrm{NH}), 140.74\left(1 \mathrm{C}, \mathrm{N}-\mathrm{C}_{\mathrm{Ar}}\right), 137.82(1 \mathrm{C}$, $\mathrm{HN}-\mathrm{C}_{\mathrm{Ar}}$ ), 136.48 (1C, Naph), 133.54 (1C, Naph), 132.94 (1C, C-Cl), 129.80 (1C, Naph), 128.96 (1C, Naph), 128.10 (1C, Naph), 127.97 (1C, Sal), 126.77 (1C, Naph), 126.22 (1C, Naph), 123.94 (1C, Naph), 123.35 (1C, Ph), 123.05 (1C, Sal), 121.98 (1C, Ph), 120.04 (1C, Ph), 119.11 (1C, Sal), 118.54 (1C, Naph), 118.00 (1C, Sal), 108.43 (1C, Ph); UV-Vis $(\mathrm{MeOH}$, $\left.3 \times 10^{-5} \mathrm{M}, \lambda / \mathrm{nm}, \varepsilon / \mathrm{cm}^{-1} \mathrm{M}^{-1}\right): 233(49,333), 264(23,333)$, 320 (16,000), 354 (16,667), 450 (12,667), 470 (12,000).

(Z)-1-(((2-((E)-(5-Bromo-2-hydroxybenzylidene) amino) phenyl) amino) methylene) naphthalen-2 $(1 \mathrm{H})$-one $\left(\mathrm{H}_{2} \mathrm{~L} 4\right)$ : yellow powder, $85 \%$; HP-TOF-MS $(\mathrm{m} / z): 445.0470$ $[M+H]^{+}(\mathrm{Cal} .446 .3160)$; IR $\left(\mathrm{KBr}, \mathrm{cm}^{-1}\right): 3061(\nu, \mathrm{C}-\mathrm{H})$, $2622(\nu, \mathrm{O}-\mathrm{H}), 1611(\nu, C=N), 1584(\nu, C=C), 1474,1351$, 1313 and $1276(\nu, \mathrm{C}-\mathrm{N}), 1179(\nu, \mathrm{C}-\mathrm{O}) ; 825,747(\delta, \mathrm{C}-\mathrm{H})$, 487; ${ }^{1}$ H-NMR (DMSO-d 6 , $\left.500 \mathrm{MHz}, \delta(\mathrm{ppm}), J(\mathrm{~Hz})\right): \delta$ $15.74(\mathrm{~d}, J=7.5,1 \mathrm{H}, \mathrm{NH}), 11.78(\mathrm{~s}, 1 \mathrm{H}, \mathrm{OH}), 9.58$ (d, $J=7.5$, $1 \mathrm{H}, \mathrm{HC}-\mathrm{N}), 8.98(\mathrm{~s}, 1 \mathrm{H}, \mathrm{HC}=\mathrm{N}), 8.44(\mathrm{~d}, J=8.5,1 \mathrm{H}, \mathrm{Naph})$, 8.28 (d, $J=2.5,1 \mathrm{H}, \mathrm{Sal}), 8.03$ (d, $J=8.0,1 \mathrm{H}, \mathrm{Naph}$ ), 7.85 (d, $J=9.0,1 \mathrm{H}, \mathrm{Naph}), 7.72(\mathrm{~d}, J=7.0,1 \mathrm{H}, \mathrm{Ph}), 7.56$ (dd, $J=8.5$, $3.0,1 \mathrm{H}, \mathrm{Sal}), 7.50(\mathrm{t}, J=7.0,1 \mathrm{H}, \mathrm{Naph}), 7.45(\mathrm{~m}, 2 \mathrm{H}, 1 \mathrm{H}-\mathrm{Ph}$, $1 \mathrm{H}-\mathrm{Naph}), 7.35(\mathrm{t}, J=7.0,1 \mathrm{H}, \mathrm{Ph}), 7.31(\mathrm{t}, 1 \mathrm{H}, \mathrm{Ph}), 6.97(\mathrm{~d}$, $J=9.0,1 \mathrm{H}, \mathrm{Sal}), 6.87(\mathrm{~d}, J=9.0,1 \mathrm{H}, \mathrm{Naph}) ;{ }^{13} \mathrm{C}-\mathrm{NMR}$ $\left(\right.$ DMSO-d $\left.\mathrm{d}_{6}, 125 \mathrm{MHz}, \delta(\mathrm{ppm})\right): \delta 175.10(1 \mathrm{C}, \mathrm{C}=\mathrm{O}), 159.97$ (1C, $C=N), 158.53$ (1C, C-O), 151.95 (1C, HC-NH), 140.72 $\left(1 \mathrm{C}, \mathrm{N}-\mathrm{C}_{\mathrm{Ar}}\right), 137.80\left(1 \mathrm{C}, \mathrm{HN}-\mathrm{C}_{\mathrm{Ar}}\right), 136.51$ (1C, Naph), 135.71 (1C, Sal), 133.54 (1C, Naph), 132.83 (1C, Sal), 128.96 (1C, Naph), 128.11 (1C, Naph), 127.98 (1C, Naph), 126.77 (1C, Ph), 126.22 (1C, Naph), 123.92 (1C, Ph), 123.35 (1C, $\mathrm{Ph}), 122.56$ (1C, Naph), 120.05 (1C, Naph), 119.12 (1C, Sal), 118.96 (1C, Naph), 118.01 (1C, Sal), 110.51 (1C, C-Br), $108.43(1 \mathrm{C}, \mathrm{Ph})$; UV-Vis $\left(\mathrm{MeOH}, 3 \times 10^{-5} \mathrm{M}, \lambda / \mathrm{nm}\right.$, $\left.\varepsilon / \mathrm{cm}^{-1} \mathrm{M}^{-1}\right): 233(44,333), 263(22,000), 320(14,333), 352$ $(14,667), 450(12,000), 472(11,000)$.

(Z)-1-(((2-((E)-(5-(Tert-butyl)-2-hydroxybenzylidene) amino) phenyl) amino) methylene) naphthalen-2 (1H)-one $\left(\mathrm{H}_{2} \mathrm{~L} 5\right)$ : yellow powder, $91 \%$. HP-TOF-MS $(\mathrm{m} / z)$ : 423.2042 $[M+H]^{+}$(Cal. 423.5262). IR $\left(\mathrm{KBr}, \mathrm{cm}^{-1}\right): 2952(\nu, \mathrm{C}-\mathrm{H})$, $2620(\nu, \mathrm{O}-\mathrm{H}), 1615(\nu, C=N), 1579(\nu, C=C), 1487,1351$, 1314 and $1288(\nu, \mathrm{C}-\mathrm{N}), 1177(\nu, \mathrm{C}-\mathrm{O}) ; 837,749(\delta, \mathrm{C}-\mathrm{H})$, 491. ${ }^{1} \mathrm{H}-\mathrm{NMR}$ (DMSO-d $\left.{ }_{6}, 500 \mathrm{MHz}, \delta(\mathrm{ppm}), J(\mathrm{~Hz})\right): \delta 15.81$ $(\mathrm{d}, J=7.5,1 \mathrm{H}, \mathrm{NH}), 11.46(\mathrm{~s}, 1 \mathrm{H}, \mathrm{OH}), 9.58(\mathrm{~d}, J=8.0,1 \mathrm{H}$, HC-N), $9.01(\mathrm{~s}, 1 \mathrm{H}, \mathrm{HC}=\mathrm{N}), 8.43(\mathrm{~d}, J=8.5,1 \mathrm{H}, \mathrm{Naph})$, 8.07 (d, $J=2.5,1 \mathrm{H}, \mathrm{Sal}), 8.02$ (d, $J=8.0,1 \mathrm{H}, \mathrm{Naph}), 7.85$ (d, $J=9.0,1 \mathrm{H}, \mathrm{Naph}), 7.71(\mathrm{~d}, J=7.0,1 \mathrm{H}, \mathrm{Ph}), 7.51-7.41(\mathrm{~m}, 4 \mathrm{H}$, $2 \mathrm{H}-\mathrm{Naph}, 1 \mathrm{H}-\mathrm{Ph}, 1 \mathrm{H}-\mathrm{Sal}), 7.35(\mathrm{t}, J=7.0,1 \mathrm{H}, \mathrm{Ph}), 7.30(\mathrm{t}$, $1 \mathrm{H}, \mathrm{Ph}), 6.92(\mathrm{~d}, J=9.0,1 \mathrm{H}, \mathrm{Sal}), 6.84(\mathrm{~d}, J=9.0,1 \mathrm{H}, \mathrm{Naph})$, $1.34(\mathrm{~s}, 9 \mathrm{H}, \mathrm{t}-\mathrm{Bu}) .{ }^{13} \mathrm{C}-\mathrm{NMR}$ (DMSO-d $, 125 \mathrm{MHz}, \delta$ $(\mathrm{ppm})): \delta 175.12(1 \mathrm{C}, \mathrm{C}=\mathrm{O}), 162.22(1 \mathrm{C}, \mathrm{C}=\mathrm{N}), 157.35(1 \mathrm{C}$, C-O), 151.88 (1C, HC-NH), 141.63 (1C, C-t-Bu), 141.29
(1C, N-C $\left.\mathrm{C}_{\mathrm{Ar}}\right), 137.77\left(1 \mathrm{C}, \mathrm{HN}-\mathrm{C}_{\mathrm{Ar}}\right), 136.34$ (1C, Naph), 133.57 (1C, Naph), 130.75 (1C, Naph), 128.98 (1C, Naph), 128.13 (1C, Sal), 127.83 (1C, Naph), 127.53 (1C, Naph), 126.85 (1C, Naph), 126.22 (1C, Ph), 123.83 (1C, Sal), 123.34 (1C, Ph), 120.02 (1C, Ph), 119.81 (1C, Naph), 119.17 (1C, Naph), 117.95 (1C, Sal), 116.13 (1C, Sal), 108.46 (1C, Ph), $33.91\left(1 \mathrm{C}, \mathrm{C}\left(\mathrm{CH}_{3}\right)_{3}, 31.21\left(3 \mathrm{H}, 3 \mathrm{CH}_{3}\right)\right.$. UV-Vis $(\mathrm{MeOH}$, $\left.3 \times 10^{-5} \mathrm{M}, \lambda / \mathrm{nm}, \varepsilon / \mathrm{cm}^{-1} \mathrm{M}^{-1}\right): 234(38,667), 266(19,333)$, 320 (14,667), 353 (14,667), 450 (12,000), 472 (11,333).

(Z)-1-(((2-((E)-(2-Hydroxy-5-methoxybenzylidene) amino) phenyl) amino) methylene) naphthalen-2 (1H)-one $\left(\mathrm{H}_{2} \mathrm{~L} 6\right)$ : yellow powder, 92\%; HP-TOF-MS $(\mathrm{m} / z): 397.1546$ $[M+H]^{+}$(Cal. 397.4459); IR $\left(\mathrm{KBr}, \mathrm{cm}^{-1}\right): 2925(\nu, \mathrm{C}-\mathrm{H})$, $2610(\nu, \mathrm{O}-\mathrm{H}), 1615(\nu, C=N), 1575(\nu, C=C), 1486,1316$ and $1273(\nu, \mathrm{C}-\mathrm{N}), 1207,1156(\nu, \mathrm{C}-\mathrm{O}) ; 1040,834,748(\delta, \mathrm{C}-\mathrm{H})$, 472; ${ }^{1}$ H-NMR (DMSO-d $\left.{ }_{6}, 500 \mathrm{MHz}, \delta(\mathrm{ppm}), J(\mathrm{~Hz})\right): \delta$ $15.81(\mathrm{~d}, J=9.0,1 \mathrm{H}, \mathrm{NH}), 10.81(\mathrm{~s}, 1 \mathrm{H}, \mathrm{OH}), 9.56(\mathrm{~d}, J=9.0$, $1 \mathrm{H}, \mathrm{HC}-\mathrm{N}), 9.03(\mathrm{~s}, 1 \mathrm{H}, \mathrm{HC}=\mathrm{N}), 8.43(\mathrm{~d}, J=8.0,1 \mathrm{H}, \mathrm{Naph})$, 8.07 (d, $J=7.5,1 \mathrm{H}, \mathrm{Naph}), 7.82$ (d, $J=9.0,2 \mathrm{H}, 1 \mathrm{H}-\mathrm{Naph}$, $1 \mathrm{H}-\mathrm{Sal}), 7.70(\mathrm{~d}, J=7.0,1 \mathrm{H}, \mathrm{Ph}), 7.51-7.41(\mathrm{~m}, 3 \mathrm{H}, 2 \mathrm{H}-$ Naph, 1H-Ph), 7.35 (t, J= 7.0,1H, Ph), $7.29(\mathrm{t}, 1 \mathrm{H}, \mathrm{Ph}), 7.03$ (dd, $J=9.0,3.5,1 \mathrm{H}, \mathrm{Sal}), 6.92$ (d, $J=9.0,1 \mathrm{H}, \mathrm{Sal}), 6.80$ (d, $J=9.0,1 \mathrm{H}, \mathrm{Naph}), 3.88\left(\mathrm{~s}, 3 \mathrm{H}, \mathrm{OCH}_{3}\right) ;{ }^{13} \mathrm{C}-\mathrm{NMR}$ (DMSO$\left.\mathrm{d}_{6}, 125 \mathrm{MHz}, \delta(\mathrm{ppm})\right): \delta 176.65(1 \mathrm{C}, \mathrm{C}=\mathrm{O}), 159.67(1 \mathrm{C}$, $C=N), 153.51(1 \mathrm{C}, \mathrm{C}-\mathrm{O}), 152.24\left(1 \mathrm{C}, \mathrm{C}-\mathrm{OCH}_{3}\right), 150.57(1 \mathrm{C}$, HC-NH), $140.81\left(1 \mathrm{C}, \mathrm{N}-\mathrm{C}_{\mathrm{Ar}}\right), 138.06\left(1 \mathrm{C}, \mathrm{HN}-\mathrm{C}_{\mathrm{Ar}}\right), 135.91$ (1C, Naph), 133.72 (1C, Naph), 128.96 (1C, Naph), 128.11 (1C, Naph), 127.59 (1C, Sal), 126.63 (1C, Naph), 126.06 (1C, Naph), 124.44 (1C, Naph), 123.26 (1C, Ph), 121.48 (1C, Sal), 120.95 (1C, Ph), 119.94 (1C, Ph), 118.65 (1C, Naph), 117.65 (1C, Naph), 117.51 (1C, Sal), 112.34 (1C, Sal), 108.20 (1C, $\mathrm{Ph}), 55.58\left(1 \mathrm{C}, \mathrm{OCH}_{3}\right.$; UV-Vis $\left(\mathrm{MeOH}, 3 \times 10^{-5} \mathrm{M}, \lambda / \mathrm{nm}\right.$, $\left.\varepsilon / \mathrm{cm}^{-1} \mathrm{M}^{-1}\right)$ : 234 (44,000), 263 (22,667), 320 (16,667), 350 $(16,667), 450(13,667), 474(12,667)$.

2.1.1. Preparation of Unsymmetrical Salen-Type Schiff Base Complexes. Unsymmetrical salen-type Schiff base complexes were prepared from the obtained ligands and $\mathrm{FeCl}_{3} \cdot 6 \mathrm{H}_{2} \mathrm{O}$ in $1: 1$ molecular ratio following the published procedure similarly [28-30]. $1.0 \mathrm{mmol} \mathrm{FeCl}_{3} \cdot 6 \mathrm{H}_{2} \mathrm{O}$ dissolved in ethanol was added to an ethanol solution of $1.0 \mathrm{mmol}$ ligand. The reaction mixture was refluxed in the presence of $1.0 \mathrm{mmol} \mathrm{Na} \mathrm{CO}_{3}$ for $3 \mathrm{hrs}$; then, the reaction mixture was cooled to room temperature. The productive precipitate was collected after being filtered and washed by cold ethanol and then dried in vacuo.

[Fe(III)L1Cl]: brown powder, 89\%; ESI-MS $(\mathrm{m} / \mathrm{z}): 419.8$ $[\mathrm{M}-\mathrm{Cl}]^{+}$(Cal. 420.2); IR (KBr, cm $\left.{ }^{-1}\right): 3054(\nu, \mathrm{C}-\mathrm{H}), 1597$ $(\nu, C=N), 1532(\nu, C=C), 1437,1378,1304(\nu, C-N), 1189(\nu$, C-O), 1140, 835, $740(\delta, \mathrm{C}-\mathrm{H}), 548(\mathrm{Fe}-\mathrm{O}), 478,456(\mathrm{Fe}-\mathrm{N})$; UV-Vis $\left(\mathrm{MeOH}, 3 \times 10^{-5} \mathrm{M}, \lambda / \mathrm{nm}, \varepsilon / \mathrm{cm}^{-1} \mathrm{M}^{-1}\right): 227$ $(44,350), 300(27,000), 334(23,350), 372(20,350), 431$ $(13,350) ; \mu_{\text {eff }}=5.94 \mathrm{BM}$.

[Fe(III)L2Cl]: brown powder, 93\%; ESI-MS $(\mathrm{m} / \mathrm{z}): 437.9$ $[\mathrm{M}-\mathrm{Cl}]^{+}$(Cal. 438.2); IR (KBr, cm $\left.{ }^{-1}\right): 3055(\nu, \mathrm{C}-\mathrm{H}), 1599$ $(\nu, C=N), 1532(\nu, C=C), 1456,1361,1303(\nu, C-N), 1185(\nu$, C-O), 1142, 826, 741 ( $\delta$, C-H), 531 (Fe-O), 497, $455(\mathrm{Fe}-\mathrm{N})$; UV-Vis $\left(\mathrm{MeOH}, 3 \times 10^{-5} \mathrm{M}, \lambda / \mathrm{nm}, \varepsilon / \mathrm{cm}^{-1} \mathrm{M}^{-1}\right): 230$ 
$(32,670), 295(18,670), 338(16,000), 369(14,350), 437$ $(9,350) ; \mu_{\mathrm{eff}}=5.78 \mathrm{BM}$.

[Fe(III)L3Cl]: brown powder, 92\%; ESI-MS $(\mathrm{m} / \mathrm{z})$ : 453.8 $[\mathrm{M}-\mathrm{Cl}]^{+}(\mathrm{Cal} .454 .6) ; \mathbf{I R}\left(\mathrm{KBr}, \mathrm{cm}^{-1}\right): 3054(\nu, \mathrm{C}-\mathrm{H}), 1597$ $(\nu, C=N), 1531(\nu, C=C), 1453,1362,1307(\nu, C-\mathrm{N}), 1188(\nu$, C-O), 1166, 828, $742(\delta, \mathrm{C}-\mathrm{H}), 552(\mathrm{Fe}-\mathrm{O}), 498,456(\mathrm{Fe}-\mathrm{N})$; UV-Vis $\left(\mathrm{MeOH}, 3 \times 10^{-5} \mathrm{M}, \lambda / \mathrm{nm}, \varepsilon / \mathrm{cm}^{-1} \mathrm{M}^{-1}\right): 233$ $(49,000), 300(24,350), 340(22,350), 373(19,350), 440$ $(13,000) ; \mu_{\mathrm{eff}}=5.86 \mathrm{BM}$.

[Fe(III)L4Cl]: brown powder, 94\%; ESI-MS $(\mathrm{m} / \mathrm{z}): 497.8$ $[\mathrm{M}-\mathrm{Cl}]^{+}(\mathrm{Cal} .499 .1) ; \mathbf{I R}\left(\mathrm{KBr}, \mathrm{cm}^{-1}\right): 3053(\nu, \mathrm{C}-\mathrm{H}), 1598$ $(\nu, C=N), 1533(\nu, C=C), 1451,1366,1308(\nu, C-N), 1187(\nu$, C-O), 827, $745(\delta, \mathrm{C}-\mathrm{H}), 534(\mathrm{Fe}-\mathrm{O}), 487,455(\mathrm{Fe}-\mathrm{N})$; UVVis $\left(\mathrm{MeOH}, 3 \times 10^{-5} \mathrm{M}, \lambda / \mathrm{nm}, \varepsilon / \mathrm{cm}^{-1} \mathrm{M}^{-1}\right): 234(48,000)$, 297 (29,350), 340 (23,000), 374 (20,000), 438 (14,000); $\mu_{\mathrm{eff}}=6.00 \mathrm{BM}$.

[Fe(III)L5Cl]: brown powder, 85\%; ESI-MS $(\mathrm{m} / \mathrm{z}): 475.9$ $[\mathrm{M}-\mathrm{Cl}]^{+}(\mathrm{Cal} .476 .3) ; \mathbf{I R}\left(\mathrm{KBr}, \mathrm{cm}^{-1}\right): 3061(\nu, \mathrm{C}-\mathrm{H}), 1600$ $(\nu, C=N), 1532(\nu, C=C), 1456,1362,1314(\nu, C-N), 1186(\nu$, C-O), 1144, 828, $741(\delta, \mathrm{C}-\mathrm{H}), 539(\mathrm{Fe}-\mathrm{O}), 484,456(\mathrm{Fe}-\mathrm{N})$; UV-Vis $\left(\mathrm{MeOH}, 3 \times 10^{-5} \mathrm{M}, \lambda / \mathrm{nm}, \varepsilon / \mathrm{cm}^{-1} \mathrm{M}^{-1}\right): 230$ $(45,670), 297(27,670), 334(23,350), 378(17,670), 436$ $(12,000) ; \mu_{\text {eff }}=5.98 \mathrm{BM}$.

[Fe(III)L6Cl]: brown powder, 87\%; ESI-MS $(\mathrm{m} / \mathrm{z}): 449.9$ $[\mathrm{M}-\mathrm{Cl}]^{+}$(Cal. 450.2); IR $\left(\mathrm{KBr}, \mathrm{cm}^{-1}\right): 3056(\nu, \mathrm{C}-\mathrm{H}), 1596$ $(\nu, C=N), 1530(\nu, C=C), 1454,1362,1300(\nu, C-N), 1190(\nu$, C-O), 826, $744(\delta$, C-H), 545 (Fe-O), 502, $456(\mathrm{Fe}-\mathrm{N})$; UVVis $\left(\mathrm{MeOH}, 3 \times 10^{-5} \mathrm{M}, \lambda / \mathrm{nm}, \varepsilon / \mathrm{cm}^{-1} \mathrm{M}^{-1}\right): 223(36,670)$, 297 (21,350), 342 (17,350), 390 (12,350), 440 (9,000); $\mu_{\text {eff }}=5.95 \mathrm{BM}$.

2.1.2. Electrochemical Studies. The electrochemical studies of all complexes were carried out using the Zahner-elektrik IM6 instrument. The cyclic voltammograms of $\mathrm{Fe}(\mathrm{III})$ complexes were recorded using $1.0 \times 10^{-3} \mathrm{M}$ concentration in acetonitrile solution and $\mathrm{LiClO}_{4} 0.1 \mathrm{M}$ as supporting electrolyte. The working electrode was a platinum electrode. The reference electrode was $\mathrm{Ag} / \mathrm{AgCl} / \mathrm{KCl}$ and platinum wire was the counterelectrode. All experiments were performed in standard electrochemical cells at room temperature at a scan rate of $100 \mathrm{mV} \mathrm{s}^{-1}$ with the potential window $-3 \mathrm{~V}$ to $+3 \mathrm{~V}$ vs. $\mathrm{Ag} / \mathrm{AgCl} / \mathrm{KCl}$ reference electrode.

2.2. In Vitro Cytotoxicity. In vitro cytotoxicity of the synthetic complexes was investigated using the modified MTT (3-(4,5-dimethylthiazol-2-yl)-2,5-diphenyl tetrazolium bromide) assay [31-34]. Briefly, human cancer cells KB (a subline of Hela tumor cell line), HepG2 (a hepatoma cell line), and a normal human cell line HEK-293 (Human Embryonic Kidney cell line) were cultured in DMEM supplemented with $10 \%$ fetal bovine serum, $100 \mu \mathrm{g} / \mathrm{mL}$ streptomycin, 100 units/mL penicillin, and $2 \mathrm{mM} \mathrm{L}$-glutamine at $37^{\circ} \mathrm{C}$ in a humidified atmosphere with $5 \% \mathrm{CO}_{2}$ and 95\% air. Approximately, 10,000 cells were cultivated in 96 plates for $24 \mathrm{hrs}$, followed by treatment with different concentrations of synthetic Fe(III) complexes in DMSO and incubated continuously for $48 \mathrm{hrs}$ more. The different concentrations of each tested complexes were 128.0, 32.0,
8.0, 2.0, and $0.5 \mu \mathrm{g} / \mathrm{ml}$. Then, tested human cells were exposed to $20 \mu \mathrm{L}$ of freshly prepared MTT $(5 \mathrm{mg} / \mathrm{ml})$ solution and incubated for $4 \mathrm{~h}$ at $37^{\circ} \mathrm{C}$ in an atmosphere of $5 \% \mathrm{CO}_{2}$. The formazan crystals obtained during MTT incubation were dissolved in $100 \mu \mathrm{L}$ of DMSO. The optical density (OD) was determined at $550 \mathrm{~nm}$ on Genios TECAN spectrophotometer. The experiments were done in triplicate for every concentration of the tested complexes. The cell viability was calculated with regard to the untreated cell control (positive control), which was set to $100 \%$ viability. The dead cell control (negative control) was set to $0 \%$ viability.

$$
\% \text { inhibition }=\frac{\mathrm{OD}(\text { positive control })-\mathrm{OD}(\text { tested complex })}{\mathrm{OD}(\text { positive control })-\mathrm{OD}(\text { negative control })} \times 100 \text {. }
$$

The percent cell inhibitions were plotted as a function of concentration to estimate the $\mathrm{IC}_{50}$ (the concentration at which a substance exerts half of its maximal inhibitory effect) values presented in Table 1 .

\section{Results and Discussion}

3.1. Synthesis and Characterization. The unsymmetrical tetradentate Schiff base ligands $\left(\mathrm{H}_{2} \mathrm{~L} 1-\mathrm{H}_{2} \mathrm{~L} 6\right)$ (Table 2) were synthesized following a two-step procedure in high yields (up to 93\%) and excellent purity (>98.7\%, Supplementary Materials). The obtained ligands are soluble in organic solvents such as DMSO, methanol, dichloromethane, and ethyl acetate. These synthetic ligands were characterized by HP-TOF-MS, IR, ${ }^{1} \mathrm{H}-\mathrm{NMR}$, and ${ }^{13} \mathrm{C}-\mathrm{NMR}$ spectroscopies. The Fe(III) complexes were prepared following the coordination of $\mathrm{FeCl}_{3} \cdot 6 \mathrm{H}_{2} \mathrm{O}$ with each obtained ligand in good yields (85-94\%) in ethanol at $\mathrm{pH} 9.0$ (Scheme 1). The synthetic unsymmetrical tetradentate Schiff base complexes are soluble in DMSO, acetonitrile, methanol, and dichloromethane. These complexes were characterized by ESI-MS, IR, UV-Vis spectroscopies, and magnetic moments $\left(\mu_{\mathrm{eff}}\right)$.

From the high-performance mass spectra, the pseudomolecular ion signals of the obtained ligands are found as $[\mathrm{M}+\mathrm{H}]^{+}$, clearly indicating molecular masses suitable for the suggested formulae. On ESI-MS spectra of synthetic unsymmetrical complexes, pseudomolecular ion peaks are assigned to $[\mathrm{M}-\mathrm{Cl}]^{-}$for $\mathrm{Fe}(\mathrm{III})$ complexes. They are in excellent agreement with the suggested formulae (Table 2).

On ${ }^{1} \mathrm{H}$-NMR spectra, usually, a typical signal at about $15.63 \mathrm{ppm}$ was found for a proton of $\mathrm{OH}$ of naphthyl group as a singlet [35], but typical signals at $15.63-15.81 \mathrm{ppm}$ as doublets, so there must be tautomerism between keto-amine and enol-imine forms here (Scheme 1) and protons of $\mathrm{NH}$ groups were found. There are typical signals at $10.81-11.93 \mathrm{ppm}$ as singlets were assigned to protons of $\mathrm{OH}$ of salicyl groups [36]. The typical signals at 9.56-9.60 ppm as doublets were probably observed for protons of $\mathrm{HC}-\mathrm{N}$ groups and at $8.98-9.03 \mathrm{ppm}$ as singlets are for protons of $\mathrm{HC}=N$ groups. The proton signals of aromatic groups were found at about $6.80-8.44 \mathrm{ppm}$. When the salicyl group containing fluor $\left(\mathrm{H}_{2} \mathrm{~L} 2\right)$ proton signals were observed as multilets as usual, there are proton signals at $1.34 \mathrm{ppm}$ as 
TABLE 1: In vitro cytotoxicity of the unsymmetrical salen-type Fe(III) complexes.

\begin{tabular}{|c|c|c|c|}
\hline \multirow{2}{*}{ Complex } & \multicolumn{3}{|c|}{$\mathrm{IC}_{50}(\mu \mathrm{M})$} \\
\hline & $\mathrm{KB}$ & HepG2 & HEK-293 \\
\hline $\mathrm{H}_{2} \mathrm{~L} 1$ & $>100$ & $51.48 \pm 2.71$ & nd \\
\hline [Fe(III)L1Cl] & $0.81 \pm 0.08$ & $3.18 \pm 0.19$ & $9.33 \pm 0.17$ \\
\hline [Fe(III)L2Cl] & $3.53 \pm 0.17$ & $25.45 \pm 1.21$ & nd \\
\hline [Fe(III)L3Cl] & $10.97 \pm 0.65$ & $63.31 \pm 2.55$ & nd \\
\hline [Fe(III)L4Cl] & $14.14 \pm 0.58$ & $56.53 \pm 2.25$ & nd \\
\hline [Fe(III)L5Cl] & $8.31 \pm 0.43$ & $28.31 \pm 1.47$ & nd \\
\hline [Fe(III)L6Cl] & $2.61 \pm 0.10$ & $1.87 \pm 0.08$ & $6.34 \pm 0.10$ \\
\hline Ellipticine & $1.14 \pm 0.08$ & $2.11 \pm 0.16$ & $6.69 \pm 0.32$ \\
\hline
\end{tabular}

nd: not determined.

TABLE 2: Unsymmetrical tetradentate Schiff base ligands and their Fe(III) complexes.

\begin{tabular}{lcr}
\hline Ligand & $\mathrm{R}$ & Fe(III) complex \\
\hline $\mathrm{H}_{2} \mathrm{~L} 1$ & $\mathrm{H}$ & [Fe(III)L1Cl] \\
$\mathrm{H}_{2} \mathrm{~L} 2$ & $\mathrm{~F}$ & [Fe(III)L2Cl] \\
$\mathrm{H}_{2} \mathrm{~L} 3$ & $\mathrm{Cl}$ & [Fe(III)L3Cl] \\
$\mathrm{H}_{2} \mathrm{~L} 4$ & $\mathrm{Br}$ & [Fe(III)L4Cl] \\
$\mathrm{H}_{2} \mathrm{~L} 5$ & $\mathrm{t}-\mathrm{Bu}$ & [Fe(III)L5Cl] \\
$\mathrm{H}_{2} \mathrm{~L} 6$ & $\mathrm{OCH}_{3}$ & [Fe(III)L6Cl] \\
\hline
\end{tabular}

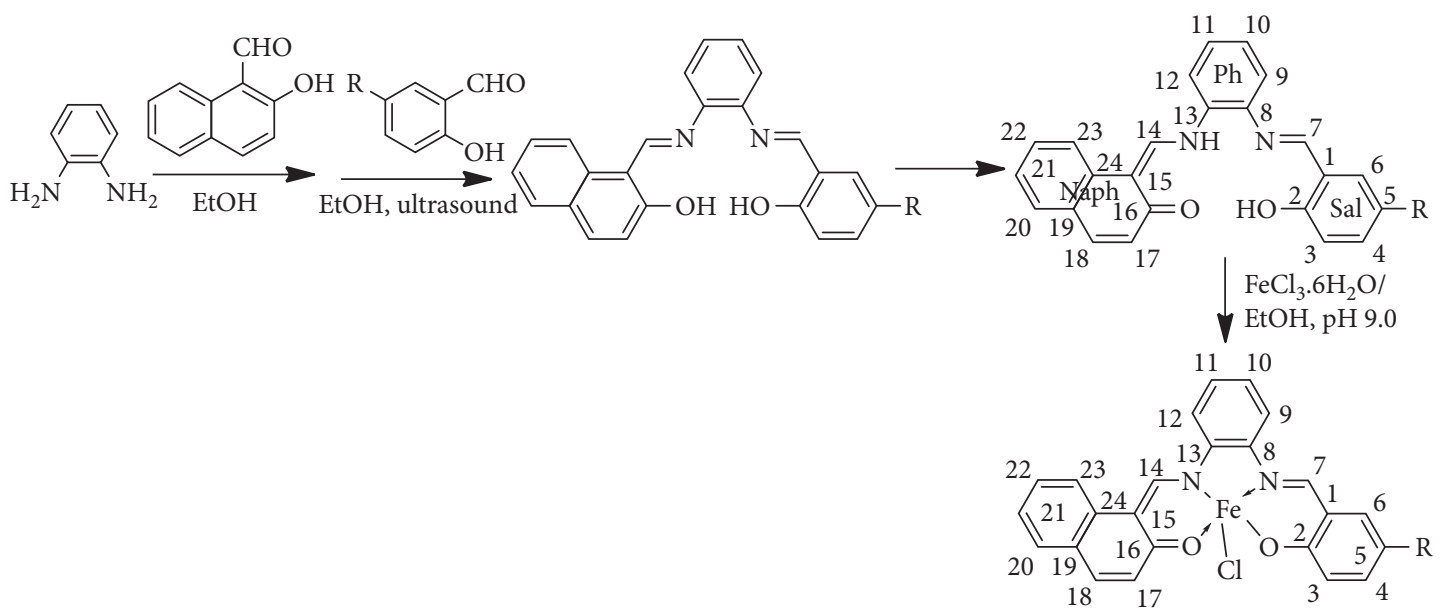

Scheme 1: Synthesis of unsymmetrical tetradentate Schiff base ligands and their Fe(III) complexes.

singlet for 9 protons of $t$-butyl and at $3.88 \mathrm{ppm}$ as singlet for 3 protons of methoxy group.

On ${ }^{13} \mathrm{C}-\mathrm{NMR}$ spectra, there are typical signals at $174.31-176.65 \mathrm{ppm}$ for $\mathrm{C}^{16}$ of $\mathrm{C}=\mathrm{O}$ groups (Scheme 1) probably. The typical signals at $159.67-162.98 \mathrm{ppm}$ were found for $\mathrm{C}^{7}$ of $C=N$ groups. The typical signals at $153.51-159.70 \mathrm{ppm}$ and $150.57-152.68 \mathrm{ppm}$ are found for the carbons of $\mathrm{C}-\mathrm{OH}$ and $\mathrm{HC}-\mathrm{N}$ groups. The typical signals at $140.72-141.29 \mathrm{ppm}$ and $137.69-138.06 \mathrm{ppm}$ were assigned to $\mathrm{N}-\mathrm{C}_{\mathrm{Ar}}$ and $\mathrm{HN}-\mathrm{C}_{\mathrm{A}}$ carbon groups. The salicyl containing fluoro group $\left(\mathrm{H}_{2} \mathrm{~L} 2\right)$ possesses the typical carbon signals in doublets. The carbon signals of $t$-butyl $\left(\mathrm{H}_{2} \mathrm{~L} 4\right)$ are found at 33.91 and $31.21 \mathrm{ppm}$ and the carbon signal of methoxy $\left(\mathrm{H}_{2} \mathrm{~L} 5\right)$ is observed at $55.58 \mathrm{ppm}$.
On IR spectra of the obtained ligands, there are typical signals of $C=N$ stretching vibrations for the formation of synthetic unsymmetrical tetradentate ligands at $1611-1618 \mathrm{~cm}^{-1}$ (Table 3 ). The typical stretching vibrations of $C=O$ bondings are found at $1540-1543 \mathrm{~cm}^{-1}$. The weak broad signals at $2715-2858 \mathrm{~cm}^{-1}$ could belong to $\mathrm{O}-\mathrm{H}$ and $\mathrm{N}-\mathrm{H}$ stretching vibrations. There are typical signals at $1273-1288 \mathrm{~cm}^{-1}$ and $1200-1211 \mathrm{~cm}^{-1}$ for C-N and C-O stretching vibrations, respectively. On IR spectra of the complexes, there are no signals for $\mathrm{O}-\mathrm{H}$ and $\mathrm{N}-\mathrm{H}$ stretching vibrations. New bands were observed for $\mathrm{Fe}-\mathrm{N}$ and $\mathrm{Fe}-\mathrm{O}$ vibrations at $531-552 \mathrm{~cm}^{-1}$ and $455-456 \mathrm{~cm}^{-1}$, respectively. They are obvious evidence for $\mathrm{H}$ separation and coordination of $\mathrm{N}$ and $\mathrm{O}$ with a metal center in the obtained 
TABLE 3: Selected typical signals of IR spectra of the obtained ligands and synthetic complexes.

\begin{tabular}{|c|c|c|c|c|c|c|c|}
\hline Compound & $\nu_{(\mathrm{O}-\mathrm{H} ; \mathrm{N}-\mathrm{H})}$ & $\nu_{(\mathrm{C}=\mathrm{N})}$ & $\nu_{(\mathrm{C}=\mathrm{O})}$ & $\nu_{(\mathrm{C}-\mathrm{N})}$ & $\nu_{(\mathrm{C}-\mathrm{O})}$ & $\nu_{(\mathrm{M}-\mathrm{N})}$ & $\nu_{(\mathrm{M}-\mathrm{O})}$ \\
\hline $\mathrm{H}_{2} \mathrm{~L} 1$ & 2715 & 1611 & 1541 & 1278 & 1211 & - & - \\
\hline [Fe(III)L1Cl] & - & 1597 & 1532 & 1252 & 1189 & 548 & 456 \\
\hline $\mathrm{H}_{2} \mathrm{~L} 2$ & 2740 & 1618 & 1542 & 1274 & 1200 & - & - \\
\hline [Fe(III)L2Cl] & - & 1599 & 1532 & 1246 & 1185 & 531 & 455 \\
\hline $\mathrm{H}_{2} \mathrm{~L} 3$ & 2780 & 1618 & 1543 & 1278 & 1211 & - & - \\
\hline [Fe(III)L3Cl] & - & 1597 & 1531 & 1260 & 1188 & 552 & 456 \\
\hline $\mathrm{H}_{2} \mathrm{~L} 4$ & 2775 & 1611 & 1542 & 1276 & 1211 & - & - \\
\hline [Fe(III)L4Cl] & - & 1598 & 1533 & 1258 & 1187 & 534 & 455 \\
\hline $\mathrm{H}_{2} \mathrm{~L} 5$ & 2858 & 1615 & 1542 & 1288 & 1210 & - & - \\
\hline [Fe(III)L5Cl] & - & 1600 & 1532 & 1256 & 1186 & 539 & 456 \\
\hline $\mathrm{H}_{2} \mathrm{~L} 6$ & 2836 & 1615 & 1540 & 1273 & 1207 & - & - \\
\hline [Fe(III)L6Cl] & - & 1596 & 1530 & 1254 & 1190 & 545 & 456 \\
\hline
\end{tabular}

complexes. The typical bands for stretching vibrations of $C=N, C=O, \mathrm{C}-\mathrm{N}$, and $\mathrm{C}-\mathrm{O}$ of $\mathrm{Fe}(\mathrm{III})$ complexes at $1596-1600 \mathrm{~cm}^{-1}, 1530-1533 \mathrm{~cm}^{-1}, 1246-1260 \mathrm{~cm}^{-1}$, and $1185-1190 \mathrm{~cm}^{-1}$, respectively. Therefore in the complexes, these bonding vibrations are shifted to a higher field than in the free ligands.

$\mathrm{UV}-\mathrm{Vis}$ spectra of the synthetic ligands and their metal complexes were measured in methanol solutions of the concentration $3.0 \times 10^{-5} \mathrm{M}$ at room temperature. UV-Vis spectra of synthetic unsymmetrical salen-type ligands were performed in Figure 1. They showed bands at 233-234 nm and $263-266 \mathrm{~nm}$, which may be assigned to the $\pi \longrightarrow \pi *$ electronic transitions of aromatic rings, at 320 and $348-355 \mathrm{~nm}$ assigned to the $n \longrightarrow \pi *$ transitions of free electrons of $\mathrm{N}$ and $\mathrm{O}$ of $\mathrm{C}=\mathrm{N}$ and $\mathrm{C}-\mathrm{O}$ [23], at 450 and $470-474 \mathrm{~nm}$ assigned to the electronic transitions of $C=O$. There are few differences in UV-Vis spectra of the ligands with different substituted groups.

$\mathrm{UV}-\mathrm{Vis}$ spectra of synthetic unsymmetrical salen-type $\mathrm{Fe}(\mathrm{III})$ complexes are obtained in Figure 2. Upon complexation, the maximum absorption bands of obtained ligands were shifted to different wavelengths, indicating the coordination of the ligands to metal [29].

The absorption bands with wavelengths of maximum absorption at $223-234 \mathrm{~nm}$ were assigned to $\pi \longrightarrow \pi *$ electronic transition of aromatic rings; $295-300 \mathrm{~nm}$ and $334-342 \mathrm{~nm}$ could present for $n \longrightarrow \pi *$ electronic transitions of free electrons on $\mathrm{N}$ and $\mathrm{O}$ of $\mathrm{C}=\mathrm{N}$ and $\mathrm{C}-\mathrm{O}$. A band is observed at 431-440 nm, which can be assigned to the electronic transitions of $C=O$. A weak band is observed at about $500 \mathrm{~nm}$, which can be assigned to LMCT transitions $[24,37]$. The $d-d$ bands were not observed due to the low concentration $\left(3.0 \times 10^{-5} \mathrm{M}\right)$ of the solutions. These bands should be of low intensity in the region of $550-650 \mathrm{~nm}$. There are also small differences in UV-Vis spectra of $\mathrm{Fe}$ (III) complexes bearing ligands with different substituted groups.

$\mathrm{Fe}(\mathrm{III})$ complexes exhibit effective magnetic moment values of $5.78-6.00 \mathrm{BM}$ due to the presence of high-spin five unpaired electrons, which indicate an octahedral geometry around $\mathrm{Fe}(\mathrm{III})$ ions $[38,39]$.

3.2. Electrochemical Studies. The electrochemical behaviors of the synthetic unsymmetrical salen-type Fe(III)

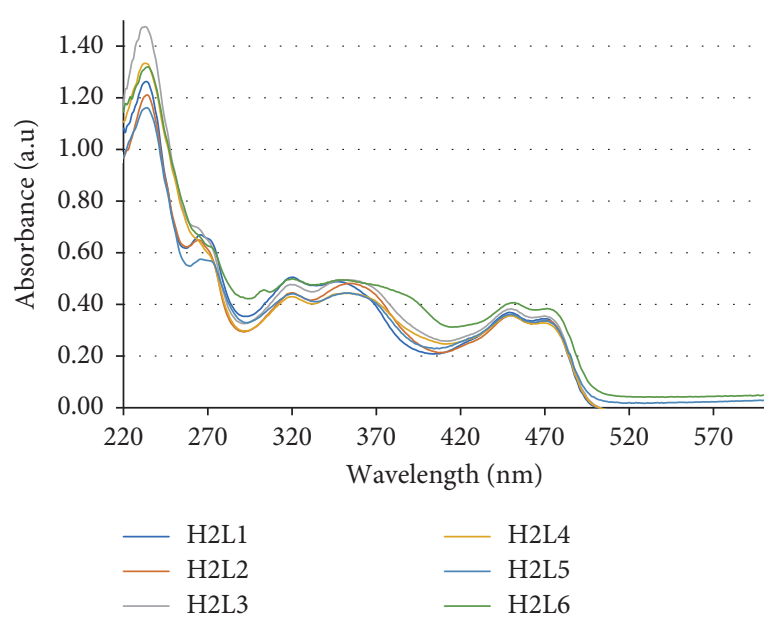

FIgURE 1: UV-Vis absorption spectra of unsymmetrical salen-type Schiff base ligands.

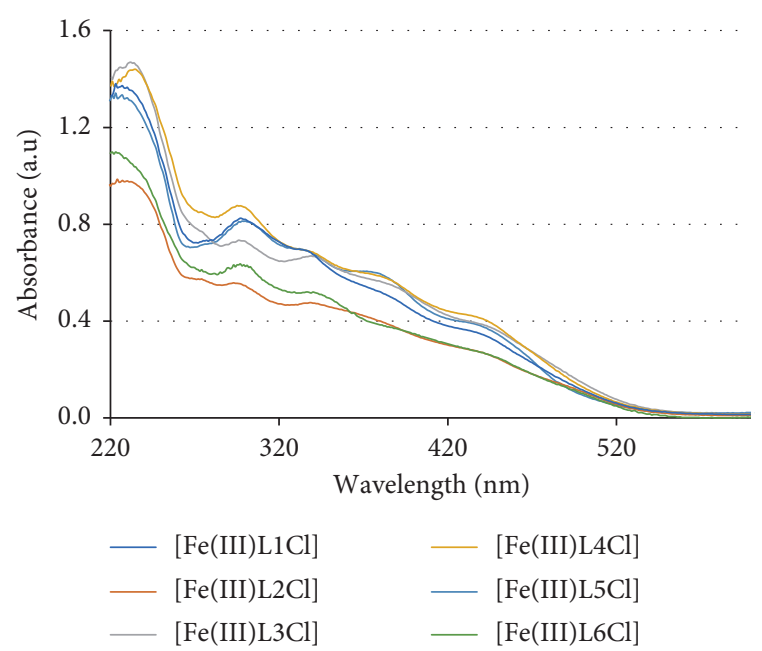

FIGURE 2: UV-Vis absorption spectra of unsymmetrical salen-type $\mathrm{Fe}(\mathrm{III})$ complexes.

complexes were studied using cyclic voltammetry (CV). Cyclic voltammograms were recorded using a Zahnerelektrik IM6 instrument with a standard three-electrode 


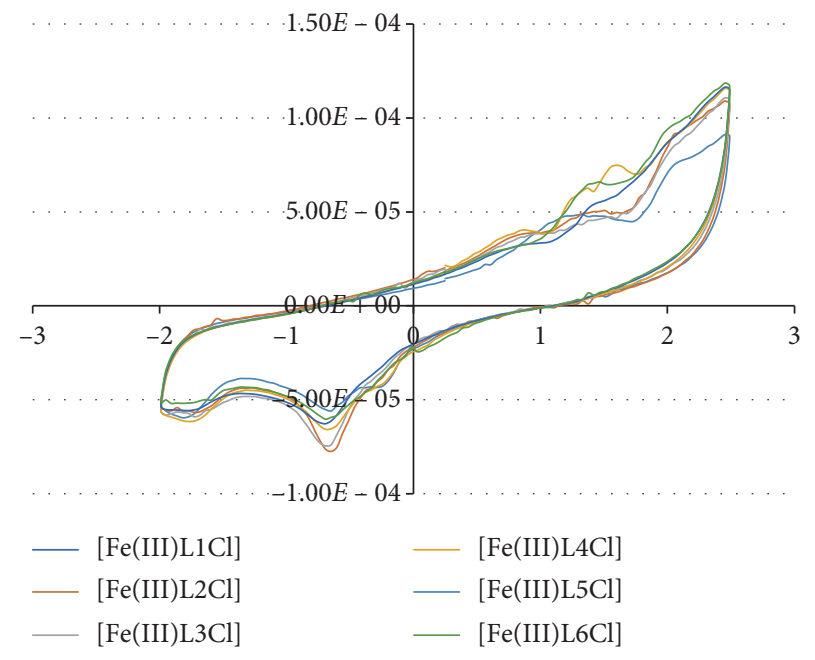

FIGURE 3: Cyclic voltammograms of unsymmetrical salen-type $\mathrm{Fe}(\mathrm{III})$ complexes.

TABLe 4: Reduction potentials of synthetic Fe(III) complexes.

\begin{tabular}{lc}
\hline Complex & Epc (V) \\
\hline$[\mathrm{Fe}(\mathrm{III}) \mathrm{L} 1 \mathrm{Cl}]$ & -0.693 \\
{$[\mathrm{Fe}(\mathrm{III}) \mathrm{L} 2 \mathrm{Cl}]$} & -0.603 \\
{$[\mathrm{Fe}(\mathrm{III}) \mathrm{L} 3 \mathrm{Cl}]$} & -0.603 \\
{$[\mathrm{Fe}(\mathrm{III}) \mathrm{L} 4 \mathrm{Cl}]$} & -0.648 \\
{$[\mathrm{Fe}(\mathrm{III}) \mathrm{L} 5 \mathrm{Cl}]$} & -0.693 \\
{$[\mathrm{Fe}(\mathrm{III}) \mathrm{L} 6 \mathrm{Cl}]$} & -0.648 \\
\hline
\end{tabular}

setup, a carbon graphite as the working electrode, a platinum wire as the counterelectrode, and $\mathrm{Ag} / \mathrm{AgCl}$ as the reference electrode, at room temperature with scan rate $=100 \mathrm{mV} \mathrm{s}^{-1}$. The concentration of complexes in acetonitrile was $1.0 \times 10^{-3} \mathrm{M}$ and $0.1 \mathrm{M} \mathrm{LiClO}_{4}$ was used as supporting electrolyte. The cyclic voltammograms of synthetic $\mathrm{Fe}(\mathrm{III})$ complexes are shown in Figure 3. Synthetic $\mathrm{Fe}(\mathrm{III})$ complexes possess well-defined cathodic peaks at (-) 0.603-(-) $0.693 \mathrm{~V}$ for irreversible reduction of $\mathrm{Fe}(\mathrm{III}) \longrightarrow \mathrm{Fe}(\mathrm{II})$ probably. A similar type of cathodic signal was observed in the reported Fe(III) complexes [40]. Some differences in the reduction potentials of the Fe(III) complexes must be expected from the electronic effects of the electron-withdrawing and electron-donating substituted groups (Table 4).

3.3. In Vitro Cytotoxicity Assay. The cytotoxicity of unsymmetrical salen-type $\mathrm{Fe}(\mathrm{III})$ complexes against two human cancer cell lines KB and HepG2 and a normal Human Embryonic Kidney cell line HEK-293 was determined by the MTT dye reduction method using ellipticine as the standard compound and the ligand $\mathrm{H}_{2} \mathrm{~L} 1$ for comparison purposes. The bioassay results are presented in Table 1 .

The results showed that the obtained $\mathrm{Fe}(\mathrm{III})$ complexes have very excellent cytotoxicity for $\mathrm{KB}$ and HepG2 with $\mathrm{IC}_{50}<15$ and $65 \mu \mathrm{M}$, respectively. The cytotoxic activity of $[\mathrm{Fe}(\mathrm{III}) \mathrm{L1Cl}]$ is much better than the one of the ligand $\mathrm{H}_{2} \mathrm{~L} 1$.
The synthetic complexes with different substituted groups possess different anticancer activity. The unsymmetrical salen-type $[\mathrm{Fe}(\mathrm{III}) \mathrm{L} 1 \mathrm{Cl}]$ and $[\mathrm{Fe}(\mathrm{III}) \mathrm{L} 6 \mathrm{Cl}]$ showed the best cytotoxic activity for $\mathrm{KB}$ and $\mathrm{HepG} 2$, respectively, with $\mathrm{IC}_{50}$ being 0.81 and $1.87 \mu \mathrm{M}$, even better than the standard compound, ellipticine, with $\mathrm{IC}_{50}=1.14$ and $2.11 \mu \mathrm{M}$ for $\mathrm{KB}$ and HepG2, respectively (Table 1 ). The cytotoxic activity order of $\mathrm{Fe}(\mathrm{III})$ complexes is indicated as [Fe(III)L1Cl] $[\mathrm{Fe}(\mathrm{III}) \mathrm{L} 6 \mathrm{Cl}]>[\mathrm{Fe}(\mathrm{III}) \mathrm{L} 2 \mathrm{Cl}]>[\mathrm{Fe}(\mathrm{III}) \mathrm{L} 5 \mathrm{Cl}]>[\mathrm{Fe}(\mathrm{III}) \mathrm{L} 3 \mathrm{Cl}]$ $\sim[\mathrm{Fe}(\mathrm{III}) \mathrm{L} 4 \mathrm{Cl}]$. In addition, [Fe(III)L1Cl] and [Fe(III)L6Cl] showed the worse cytotoxic activity in several times for the normal human cell line HEK-293 with $\mathrm{IC}_{50}$ being 9.33 and $6.34 \mu \mathrm{M}$, respectively. This selectivity is quite good and similar to the selectivity of ellipticine with $\mathrm{IC}_{50}=6.69 \mu \mathrm{M}$ for HEK-293.

\section{Conclusions}

In this study, we have reported the synthesis and characterization of novel $\mathrm{Fe}$ (III) complexes bearing various unsymmetrical salen-type ligands. The ligands with electron-donating and electron-withdrawing substituted groups have some effects on their spectral properties. The UV-Vis absorption bands for LMCT of the Fe(III) complexes were observed at $430-440 \mathrm{~nm}$. Interestingly, the cyclic voltammograms of the Fe(III) complexes show cathodic peaks for irreversible reduction of $\mathrm{Fe}(\mathrm{III}) \longrightarrow$ $\mathrm{Fe}(\mathrm{II})$ at $(-)$ 0.603-(-) $0.693 \mathrm{~V}$. The obtained Fe(III) complexes were all estimated on the cytotoxicity in vitro for KB and HepG2 human cancer cells. The results showed that the synthetic Fe(III) complexes have excellent cytotoxicity for KB and HepG2 human cancer cells ( $\mathrm{IC}_{50}<15$ and $65 \mu \mathrm{M}$, respectively). Among them, $[\mathrm{Fe}(\mathrm{III}) \mathrm{L} 1 \mathrm{Cl}]$ and $[\mathrm{Fe}(\mathrm{III}) \mathrm{L} 6 \mathrm{Cl}]$ showed the best cytotoxic activity for $\mathrm{KB}$ and HepG2 with $\mathrm{IC}_{50}=0.81$ and $1.87 \mu \mathrm{M}$, respectively, even better than the standard compound, ellipticine, with $\mathrm{IC}_{50}=1.14$ and $2.11 \mu \mathrm{M}$ for $\mathrm{KB}$ and HepG2 respectively.

\section{Data Availability}

All data used to support this report's findings are included within the article and in supplementary materials.

\section{Conflicts of Interest}

The authors declare that they have no conflicts of interest.

\section{Acknowledgments}

This research was financially supported by Vietnam NAFOSTED (grant no. 104.01-2018.366).

\section{Supplementary Materials}

The spectral data of synthetic unsymmetrical salen-type ligands and their Fe(III) complexes. (Supplementary Materials) 


\section{References}

[1] W.-H. Zhang, S. W. Chien, and T. S. A. Hor, "Recent advances in metal catalysts with hybrid ligands," Coordination Chemistry Reviews, vol. 255, no. 17-18, pp. 1991-2024, 2011.

[2] C. Zhong and X. Shi, "When organocatalysis meets transitionmetal catalysis," European Journal of Organic Chemistry, vol. 16, pp. 2999-3025, 2010.

[3] N.-F. Mazlan, L. L. Tan, N. H. A. Karim, L. Y. Heng, and M. I. H. Reza, "Optical biosensing using newly synthesized metal salphen complexes: a potential DNA diagnostic tool," Sensors and Actuators B: Chemical, vol. 242, pp. 176-188, 2017.

[4] P. K. Sonkar and V. Ganesan, V. Rao, "Electrocatalytic oxidation and determination of cysteine at oxovanadium (IV) salen coated electrodes," International Journal of Electrochemical Science, vol. 2014, Article ID 316254, 6 pages, 2014.

[5] R. E. Demirdogen, D. Kilic, F. M. Emen et al., "Novel antibacterial cellulose acetate fibers modified with 2-fluoropyridine complexes," Journal of Molecular Structure, vol. 1204, Article ID 127537, 2020.

[6] Q.-C. Zhang, H. Xiao, X. Zhang, L.-J. Xu, and Z.-N. Chen, "Luminescent oligonuclear metal complexes and the use in organic light-emitting diodes," Coordination Chemistry Reviews, vol. 378, pp. 121-133, 2019.

[7] R. E. Mewis and S. J. Archibald, "Biomedical applications of macrocyclic ligand complexes," Coordination Chemistry Reviews, vol. 254, no. 15-16, pp. 1686-1712, 2010.

[8] C.-N. Ko, G. Li, C.-H. Leung, and D.-L. Ma, "Dual function luminescent transition metal complexes for cancer theranostics: the combination of diagnosis and therapy," Coordination Chemistry Reviews, vol. 381, pp. 79-103, 2019.

[9] S. Sharma, M. Chauhan, A. Jamsheera et al., "Chiral transition metal complexes: synthetic approach and biological applications," Inorganica Chimica Acta, vol. 458, no. 8-27, 2017.

[10] J. G. Pereda, Á. Martínez, A. Terenzi et al., "Anticancer platinum agents and light," Inorganica Chimica Acta, vol. 495, Article ID 118981, 2019.

[11] M. Imran, W. Ayub, I. S. Butler, and Z.-U. Rehman, "Photoactivated platinum-based anticancer drugs," Coordination Chemistry Reviews, vol. 376, pp. 405-429, 2018.

[12] H. Chen and M. Liu, "Synthesis, crystal structure and in vitro anticancer studies of two bis (8-quinolinolato-N,O)-platinum (II) complexes," European Journal of Chemistry, vol. 10, no. 1, pp. 37-44, 2019.

[13] M. T. Kaczmarek, M. Zabiszak, M. Nowak, and R. Jastrzab, "Lanthanides: schiff base complexes, applications in cancer diagnosis, therapy, and antibacterial activity," Coordination Chemistry Reviews, vol. 370, pp. 42-54, 2018.

[14] M. S. More, P. G. Joshi, Y. K. Mishra, and P. K Khanna, "Metal complexes driven from Schiff bases and semicarbazones for biomedical and allied applications: a review," Materials Today. Chemistry, vol. 14, Article ID 100195, 2019.

[15] Â. De Fátima, C. D. P. Pereira, C. R. S. D. G. Olímpio, B. G. De Freitas Oliveira, L. L. Franco, and P. H. C. Da Silva, "Schiff bases and their metal complexes as urease inhibitors-a brief review," Journal of Advanced Research, vol. 13, pp. 113-126, 2018.

[16] C. M. da Silva, M. M. Silva, F. S. Reis et al., "Studies on free radical scavenging, cancer cell antiproliferation, and calf thymus DNA interaction of schiff bases," Journal of Photochemistry and Photobiology B: Biology, vol. 172, pp. 129-138, 2017.
[17] C. Freire, M. Nunes, C. Pereira, D. M. Fernandes, A. F. Peixoto, and M. Rocha, "Metallo (salen) complexes as versatile building blocks for the fabrication of molecular materials and devices with tuned properties," Coordination Chemistry Reviews, vol. 394, pp. 104-134, 2019.

[18] V. Z. Mota, G. S. G. de Carvalho, P. P. Corbi et al., "Crystal structure and theoretical studies of the keto-enol isomerism of N,N'-bis (salicylidene)-o-phenylenediamine (salophen)," Spectrochimica Acta Part A: Molecular and Biomolecular Spectroscopy, vol. 99, pp. 110-115, 2012.

[19] A. Erxleben, "Transition metal salen complexes in bioinorganic and medicinal chemistry," Inorganica Chimica Acta, vol. 472, pp. 40-57, 2018.

[20] J. C. Pessoa and I. Correia, "Salan vs. salen metal complexes in catalysis and medicinal applications: virtues and pitfalls," Coordination Chemistry Reviews, vol. 388, pp. 227-247, 2019.

[21] S. O. Bahaffi, A. A. Abdel Aziz, and M. M. El-Naggar, "Synthesis, spectral characterization, DNA binding ability and antibacterial screening of copper (II) complexes of symmetrical NOON tetradentate Schiff bases bearing different bridges," Journal of Molecular Structure, vol. 1020, pp. 188196, 2012.

[22] G. Ahumada, M. Fuentealba, T. Roisnel et al., "Novel Co (II), $\mathrm{Ni}$ (II) and $\mathrm{Cu}$ (II) complexes involving a 2-thienyl and trifluoromethyl containing symmetrically-substituted tetradentate Schiff-base ligand: syntheses, structures, electrochemical and computational studies," Polyhedron, vol. 151, pp. 279-286, 2018.

[23] Q. T. Nguyen, P. N. Pham Thi, and V. T. Nguyen, "Synthesis, characterization, and in vitro cytotoxicity of platinum (II) complexes bearing chiral tetradentate salicylaldimine ligands," Journal of Chemistry, vol. 2020, Article ID 5414959, 10 pages, 2020.

[24] S. N. Shukla, P. Gaur, M. L. Raidas et al., "Tailored synthesis of unsymmetrical tetradentate ONNO schiff base complexes of Fe (IIl), Co (II) and Ni (II): spectroscopic characterization, DFT optimization, oxygen-binding study, antibacterial and anticorrosion activity," Journal of Molecular Structure, vol. 1202, Article ID 127362, 2020.

[25] M. A. Said, A. Al-unizi, M. Al-Mamary et al., "Easy coordinate geometry indexes, $\tau 4$ and $\tau 5$ and HSA study for unsymmetrical Pd (II), Fe (II), Zn (II), Mn (II), Cu (II) and VO (IV) complexes of a tetradentate ligand: synthesis, characterization, properties, and antioxidant activities," Inorganica Chimica Acta, vol. 505, Article ID 119434, 2020.

[26] S. Meghdadi, M. Amirnasr, M. Majedi et al., "Template synthesis, and X-ray crystal structures of copper (II) and nickel (II) complexes of new unsymmetrical tetradentate Schiff base ligands. Electrochemistry, antibacterial properties, and metal ion effect on hydrolysis-recondensation of the ligand," Inorganica Chimica Acta, vol. 437, pp. 64-69, 2015.

[27] K. Ambroziak and M. Szypa, "A synthesis of unsymmetrical chiral salen ligands derived from 2-hydroxynaphthaldehyde and substituted salicylaldehydes," Tetrahedron Letters, vol. 48, no. 19, pp. 3331-3335, 2007.

[28] Q. T. Nguyen, P. N. Pham Thi, and V. T. Nguyen, "Synthesis, characterization, and in vitro cytotoxicity of unsymmetrical tetradentate schiff base Cu (II) and Fe (III) complexes," Bioinorganic Chemistry and Applications, vol. 2021, Article ID 6696344, 10 pages, 2021.

[29] D. Çakmak, S. Çakran, S. Yalçinkaya, and C. Demetgül, "Synthesis of salen-type Schiff base metal complexes, electropolymerization on graphite electrode surface and investigation of electrocatalytic 
effects," Journal of Electroanalytical Chemistry, vol. 808, pp. 65-74, 2018.

[30] I. P. Ejidike and P. A. Ajibade, "Synthesis, characterization, antioxidant, and antibacterial studies of some metal (II) complexes of tetradentate schiff base ligand: (4e)-4-[(2-\{(E)[1-(2,4- dihydroxyphenyl) ethylidene] amino\} ethyl) imino] pentan-2-one," Bioinorganic Chemistry and Applications, vol. 2015, Article ID 890734, , 2015.

[31] T. Mosmann, "Rapid colorimetric assay for cellular growth and survival: application to proliferation and cytotoxicity assays," Journal of Immunological Methods, vol. 65, no. 1-2, pp. 55-63, 1983.

[32] F. Denizot and R. lang, "Rapid colorimetric assay for cell growth and survival," Journal of Immunological Methods, vol. 89, no. 2, pp. 271-277, 1986.

[33] G. Fotakis and J. A. Timbrell, "In vitro cytotoxicity assays: comparison of LDH, neutral red, MTT and protein assay in hepatoma cell lines following exposure to cadmium chloride," Toxicology Letters, vol. 160, no. 2, pp. 171-177, 2006.

[34] J. Vančo, Z. Šindelář, Z. Dvořák, and Z. Trávníček, "Ironsalophen complexes involving azole-derived ligands: a new group of compounds with high-level and broad-spectrum in vitro antitumour activity," Journal of Inorganic Biochemistry, vol. 142, pp. 92-100, 2015.

[35] A. A. Nejo, G. A. Kolawole, A. R. Opoku, C. Muller, and J. Wolowska, "Synthesis, characterization, and insulin-enhancing studies of unsymmetrical tetradentate Schiff-base complexes of oxovanadium (IV)," Journal of Coordination Chemistry, vol. 62, no. 21, pp. 3411-3424, 2009.

[36] A. Hille and R. Gust, "Influence of methoxy groups on the antiproliferative effects of [FeIII (salophene-OMe) $\mathrm{Cl}$ ] complexes," European Journal of Medicinal Chemistry, vol. 45, no. 11, pp. 5486-5492, 2010.

[37] D. Das, M. K. Raza, and T. K. Goswami, "Evaluation of photochemotherapeutic potential of a few oxo-bridged dimeric Fe (III) compounds having salen-type ligands," Polyheron, vol. 186, Article ID 114614, 2020.

[38] T. Basak, A. Frontera, and S. Chattopadhyay, "Existence of strong $\mathrm{C}-\mathrm{H} \bullet \bullet \pi$ (chelatering) interaction compared to $\mathrm{C}-\mathrm{H} \bullet \bullet \pi$ (arene) interaction in the supramolecualar assembly of dinuclear iron (III) Schiff base complexes: a theoretical insight," Inorganica Chimica Acta, vol. 516, Article ID 120081, 2021.

[39] X. Liu, C. Manzur, N. Novoa, S. Celedón, D. Carrillo, and J.-R. Hamon, "Multidentate unsymmetrically-substituted Schiff bases and their metal complexes: synthesis, functional materials properties, and applications to catalysis," Coordination Chemistry Reviews, vol. 357, pp. 144-172, 2018.

[40] M. V. N. Raj, K. Bhar, S. Jain, M. Rana, T. A. Khan, and A. K. Sharma, "Syntheses, X-ray structures, electrochemical properties and biological evaluation of mono- and dinuclear N2O2-donor ligand-Fe systems," Transition Metal Chemistry, vol. 44, no. 7, pp. 615-626, 2019. 